AlSaadi et al., Afr J Tradit Complement Altern Med., (2018) 15 (2): 35-41

https://doi.org/10.21010/ajtcam.v15i2.5

\title{
HEPATOPROTECTIVE ACTIVITY OF COSTUS SPECIOSUS (KOEN. EX. RETZ.) AGAINST PARACETAMOL-INDUCED LIVER INJURY IN MICE
}

\author{
Baiaan H. AlSaadi ${ }^{1}$, Shoaa H. AlHarbi ${ }^{1}$, Sabrin R. M. Ibrahim ${ }^{2 *}$, Amal A. El-Kholy ${ }^{3,4}$, Dina S. El- \\ Agamy $^{5,6}$, and Gamal A. Mohamed
}

${ }^{1}$ Department of Pharmacy and Pharmaceutical Sciences, College of Pharmacy, Taibah University, Al Madinah Al Munawwarah 30078, Saudi Arabia; ${ }^{2}$ Department of Pharmacognosy and Pharmaceutical Chemistry, College of Pharmacy, Taibah University, Al Madinah Al Munawwarah 30078, Saudi Arabia; ${ }^{3}$ Department of Clinical and Hospital Pharmacy, College of Pharmacy, Taibah University, Al Madinah Al Munawwarah 30078, Saudi Arabia; ${ }^{4}$ Department of Clinical Pharmacy, Faculty of Pharmacy, Ain-Shams University, Cairo 11566, Egypt; ${ }^{5}$ Department of Pharmacology, College of Pharmacy, Taibah University, Al Madinah Al Munawwarah 30078, Saudi Arabia; ${ }^{6}$ Department of Pharmacology, Faculty of Pharmacy, Mansoura University, Mansoura 35516, Egypt; ${ }^{7}$ Department of Natural Products and Alternative Medicine, Faculty of Pharmacy, King Abdulaziz University, Jeddah 21589, Saudi Arabia

*Corresponding Author's E-mail: sribrahim@taibahu.edu.sa; $\underline{\text { sabrinshaur@gmail.com }}$

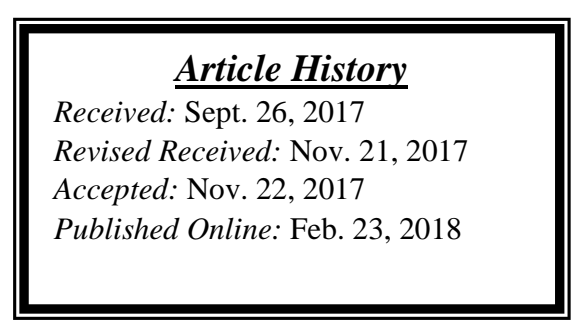

\begin{abstract}
Background: Liver diseases are a common cause of mortality and morbidity over the world. It is caused mainly by toxic chemicals and chemotherapeutic agents. Costus speciosus (Koen ex. Retz.) (Zingiberaceae) is widely employed in various traditional medicines for the prevention and treatment of different aliments. The purpose of this study is to assess the protective effect of $C$. speciosus rhizomes $\mathrm{MeOH}$ extract against the injury of the liver induced by paracetamol (PA) in mice.

Material and Methods: The mice were pretreated for seven days with distilled $\mathrm{H}_{2} \mathrm{O}$, silymarin $12 \mathrm{mg} / \mathrm{kg}$ or 100 and $200 \mathrm{mg} / \mathrm{kg} \mathrm{MeOH}$ extract. Then, PA $(750 \mathrm{mg} / \mathrm{kg}$ ) was also intra-peritoneal administrated once a day. Animals were euthanatized $24 \mathrm{~h}$ after the damage inducement. The levels of the serum enzymes aspartate aminotransferase (AST), alkaline phosphatase (ALP), alanine aminotransferase (ALT), and aspartate aminotransferase, in addition to the tumor necrosis factor-alpha (TNF- $\alpha$ ), were determined. Moreover, the histopathological examination was carried out.

Results: Administration of the $\mathrm{MeOH}$ extract $(200 \mathrm{mg} / \mathrm{kg})$ showed improvement in the toxic effects of PA through significant fall on the serum markers enzymes of liver damage: AST, ALT, and ALP, as well as TNF- $\alpha$, compared to silymarin. In parallel, the histopathological profile in the mice' liver also proved that extract markedly minimized the PA toxicity and maintained the liver tissues` histoarchitecture to near the normal ones more than that achieved by silymarin.

Conclusion: The findings suggested that $C$. speciosus extract acts as a potential hepatoprotective agent against PAinduced liver toxicity. This hepato-protection effect may be due to the existence of steroids, saponins, different glycosides, and phenolic compounds in C. speciosus.
\end{abstract}

Keywords: Costus speciosus (Koen ex. Retz.), Zingiberaceae, Hepatoprotective activity, Paracetamol

\section{Introduction}

The liver is the fundamental organ for the metabolism and excretion. It performs a vital function in detoxifying environmental pollutants, xenobiotics, and chemotherapeutic agents. Liver disease is a common cause of mortality and morbidity over the world and mainly caused by toxic chemicals, such as carbon tetrachloride and chemotherapeutic agents (Maheswari et al., 2008). Paracetamol (PA) is extensively utilized for its antipyretic and analgesic effects at therapeutic doses (Hazai et al., 2001). An overdose causes severe nephrotoxicity and hepatotoxicity (Vermeulen et al., 1992). Available pharmaco-therapeutic options for liver diseases are very limited and there is a great need for the development of safe and effective drugs. Several researches have shown that the extracts of plant possessing antioxidant activity, that protects against PA hepatotoxicity by promoting the activity of antioxidant enzyme 
and suppressing peroxidation of lipid (Cohen and Khairallah, 1997). The family Costaceae (Zingiberaceae) is divided into $\approx 52$ genera and includes about 1,300 species (Lijuan et al., 2011; Singh et al., 2011). It is distributed in various countries throughout tropical Africa, Asia, and America (Lijuan et al., 2011; Singh et al., 2011; Gupta, 2001). Costus speciosus (Koen ex. Retz.) has been considered to be a medicinal and ornamental plant and is mainly cultivated in India. It has a number of traditional uses, which are known in various countries, as well as in Saudi Arabia, such as bitter, astringent, expectorant, tonic, purgative, acrid, stimulant, depurative, anthelmintic, and expectorant (Gupta, 2010; Chopra et al., 2006). In addition, its rhizomes juice is applied for cooling on the head and remove headache. Also, rhizomes are used in anemia, asthma, bronchitis, leprosy, jaundice, and skin and urinary diseases (Sivarajan and Balachandran, 1994). The rhizomes alkaloid extract has antispasmodic and papaverine-like smooth muscle-relaxant effects (Lijuan et al., 2011; Banerji et al., 1982; Bhattacharya et al., 1972). Its extract is also used to control syphilis in Japanese traditional medicine (Jain, 1991). C. speciosus possessed various activities: antibacterial, antifungal (Ibrahim et al. 2017), anti-inflammatory, analgesic, antihyperglycemic, antihyperlipidemic, antipyretic, and antioxidant (Bavarva and Narasimhacharya, 2008; Srivastava et al., 2013). Moreover, C. speciosus saponins had antifertility and oestrogenic activities (Tewari et al., 1973). Former phytochemical studies on C. speciosus resulted in the separation of sesquiterpenes (Ibrahim et al., 2017, Al-Attas et al., 2015), sterols (Akhila et al., 1987), saponins (Ichinose et al., 1999; Inoue and Ebizuka, 1996), phenolics (Chang et al., 2012), benzoquinones (Mahmood et al., 1984), fatty acids (Gupta et al., 1986), and alkaloids (Bhattacharya et al., 1973).

In this work, the hepatoprotective potential of $C$. speciosus rhizomes was estimated against PA-induced acute liver damage. Liver enzymes serum levels alanine aminotransferase (ALT), aspartate aminotransferase (AST), and alkaline phosphatase (ALP) were estimated as the biochemical markers of liver function. In addition to the tumor necrosis factor-alpha (TNF- $\alpha$ ), was determined. Additionally, the histopathology of the liver was observed microscopically for any PA-induced toxic changes.

\section{Materials and Methods Chemicals}

AST, ALT, ALP, and TNF- $\alpha$ kits were procured from Salima Trading Corporation, Riyadh, Saudi Arabia. Paracetamol and silymarin were purchased from Sedico and EPICO Pharmaceutical Co. (Cairo, Egypt), respectively.

\section{Plant material and extract preparation}

The dried rhizomes of $C$. speciosus (Koen ex. Retz.) for the present investigation were obtained from a Saudi local market (Al-Madinah Al-Munawwarah) and authenticated by Prof Dr. Abdelaziz Fayed, Professor (Faculty of Science, Assiut University, Egypt). A specimen (CS-6-2016) was kept at the Pharmacognosy and Pharmaceutical Chemistry Department, Faculty of Pharmacy, Taibah University, KSA. A powdered sample of rhizomes (150 g) was extracted, using $\mathrm{MeOH}(4 \times 500 \mathrm{~mL})$. The extract was concentrated under vacuum, using a rotary evaporator (Heidolph, Schwabach, Germany) to give a brown residue $(19 \mathrm{~g})$.

\section{Animals and experimental design}

Adult male albino mice (20-22 g) were obtained from Department of Pharmacology, Faculty of Pharmacy, King Abdulaziz University. The mice were housed in polypropylene cages and kept at $25 \pm 2^{\circ}$ under $14 / 10 \mathrm{~h}$ dark and light cycle. They were provided with food and water ad libitum. Experiments on the animals were approved by the Research Ethics Committee of the College of Pharmacy, Taibah University. The animals selected for the present investigation were separated into five groups of 6 animals each. Group 1 (control group), was treated with distilled water as a vehicle for seven days. Group 2 was treated with paracetamol $(750 \mathrm{mg} / \mathrm{kg}$ b.w.) once a day intra-peritoneal (IP) (Steinebrunner et al., 2014). Groups 3, 4, and 5 received orally the MeOH extract (100 and $200 \mathrm{mg} / \mathrm{kg}$ b.w.) and silymarin (12 mg/kg b.w.) (Elizabeth et al., 1996), respectively once a day for seven days followed by a single IP administration of PA (750 mg/kg b.w.), 24 hours after receiving the final dose.

\section{Biochemical parameters}

After 12 hours of receiving their last treatment dose, all of the mice were anesthetized by ketamine injection and sacrificed by cervical dislocation. Samples of blood were obtained by cardiac puncture and instantly placed into ice-chilled disposable siliconized glass tubes. Centrifugation of the blood samples was carried out at $4000 \mathrm{rpm}$ at $4^{\circ} \mathrm{C}$ for $15 \mathrm{~min}$ to get serum, which was kept at $-80{ }^{\circ} \mathrm{C}$ for assessing serum enzyme levels. The liver of animals for each group was dissected, weighed, and preserved in the formalin solution for histopathological study.

\section{Histopathological study}

The livers were rinsed with ice-cold saline and cross-sectioned. Ten percent neutral buffered formalin was used for saving liver specimens. The specimens were embedded in paraffin to prepare for sectioning (4-5 $\mu \mathrm{m})$ and 
subjected to eosin and hematoxylin $(\mathrm{E} \& \mathrm{H})$ staining. Finally, the specimens were investigated under a microscope (Olympus CX-21, Shanghai Selon Scientific Instrument Co., Ltd., Shanghai, China).

\section{Statistical analysis}

The data were expressed as a mean \pm standard error of the mean (SEM). One-way analysis of variance (ANOVA) was used to analyze the data, followed by Student's $t$-test. A $p$-value of less than 0.001 was considered to be statistically significant when compared to the control.

\section{Results and Discussion}

As shown in Fig. 1-4, the serum enzyme parameter levels (AST, ALT, ALP, and TNF- $\alpha$ ) were significantly increased in the PA-treated group.

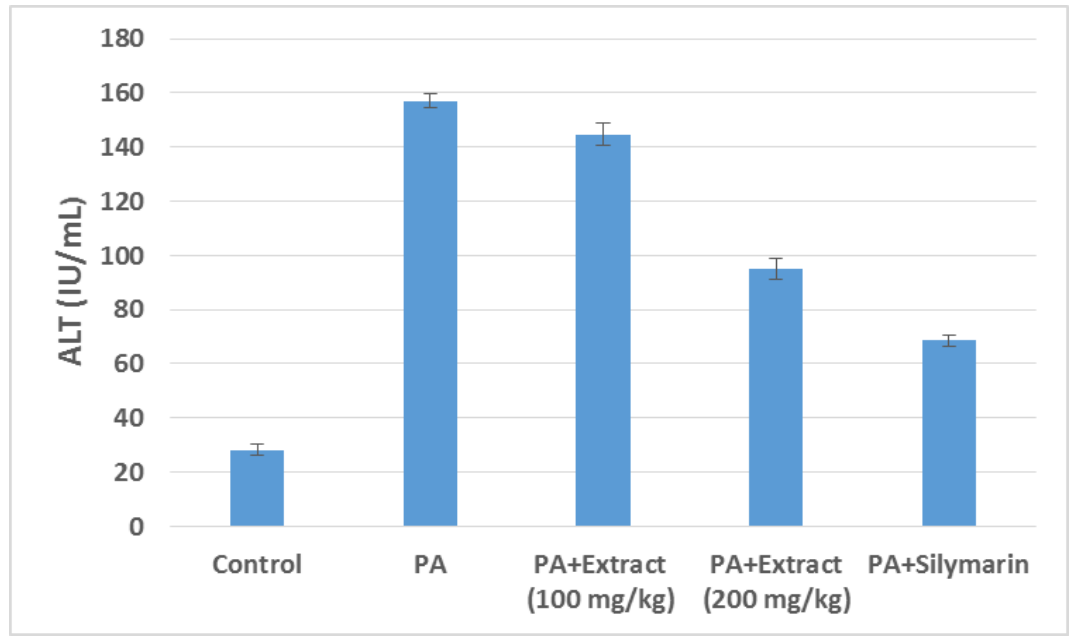

Figure :: Level of alanine aminotransferase (ALT) enzyme

A mean \pm standard error of the mean was used to express the data $(n=6)$. Group 2 (PA group) $(p=<0.001)$ was compared to group 1 (control group). Groups 3 and 4 ( $p=<0.001$ ) were compared to group 2 (one-way analysis of variance was applied, followed by Student's $t$-test).

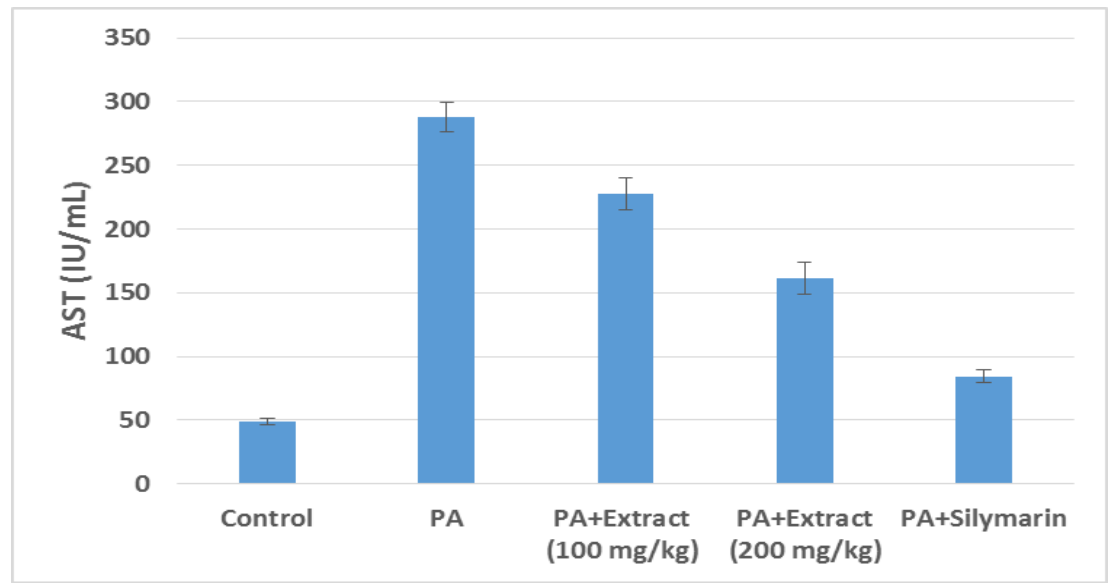

Figure 2: Level of aspartate aminotransferase (AST) enzyme

Mean \pm standard error of the mean was used to express the data $(n=6)$. Group 2 (PA group) $(p=<0.001)$ was compared to group 1 (control group). Groups 3 and $4(p=<0.001)$ were compared to group 2 (one-way analysis of variance was applied, followed by Student's $t$-test). 


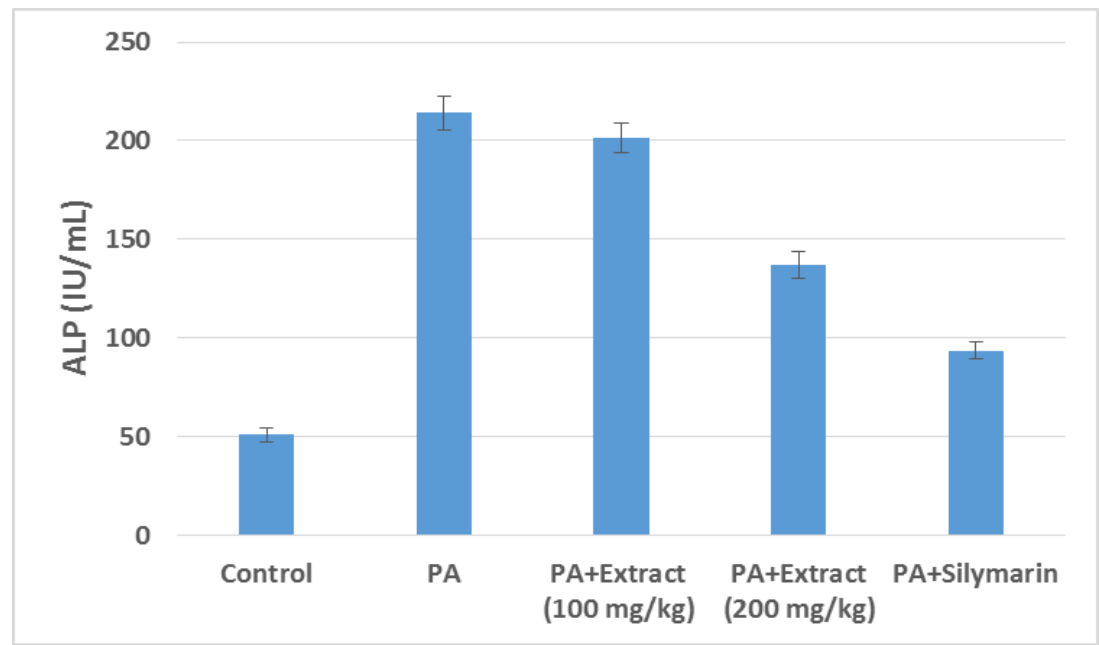

Figure 3: Level of alkaline phosphatase (ALP) enzyme

Mean \pm standard error of the mean was used to express the data $(n=6)$. Group 2 (PA group) $(p=<0.001)$ was compared to group 1 (control group). Groups 3 and $4(p=<0.001)$ were compared to group 2 (one-way analysis of variance was applied, followed by Student's $t$-test).

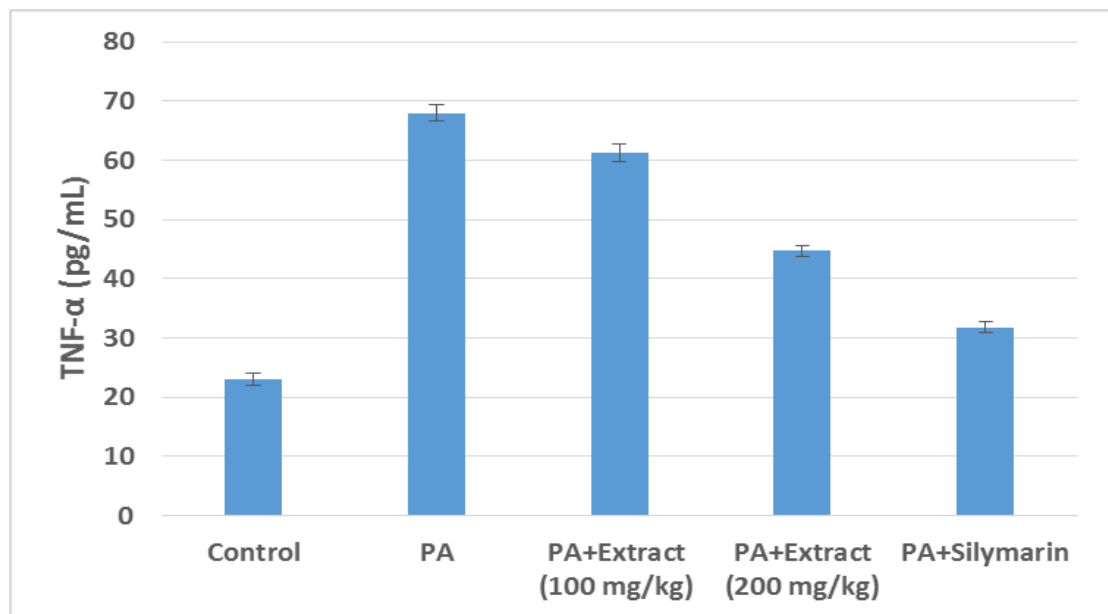

Figure 4: Level of tumor necrosis factor-alpha (TNF- $\alpha)$

Mean \pm standard error of the mean was used to express the data $(n=6)$. Group 2 (PA group) $(p=<0.001)$ was compared to group 1 (control group). Groups 3 and 4 ( $p=<0.001)$ were compared to group 2 (one-way analysis of variance was applied, followed by Student's $t$-test).

Histopathological examination of the liver tissue of mice received distilled water (normal control group) showed apparently normal hepatic tissue. Where the hepatic cells are radially placed and each cell has a granular cytoplasm and large spherical nucleus without any injury (Fig. 5A). However, liver tissues of PA-treated mice revealed congestion of the liver cells, necrosis, and apoptosis (Fig. 5B). Treatment with $C$. speciosus rhizomes $\mathrm{MeOH}$ extract at a dose $100 \mathrm{mg} / \mathrm{kg}$ did not significantly decrease AST, ALT, ALP, and TNF- $\alpha$ levels compared to silymarin. In the parallel to biochemical observation, the histopathological studies demonstrated a mild improvement in the liver structure (Fig. 5C). At extract dose $200 \mathrm{mg} / \mathrm{kg}$, the serum level of AST, ALT, ALP, and TNF- $\alpha$ was significantly decreased. Interestingly, the histopathological profile at a dose $200 \mathrm{mg} / \mathrm{kg}$ showed no significant fatty changes, hepatic inflammation, or hepatocyte necrosis were observed, which means a significant improvement in the liver structure more so than that achieved using silymarin with respect to PA-induced necrosis in the normal compact arrangement of the liver cells in animals (Fig. 5D\&E). The search for safe and effective medication to treat hepatic disorders is of considerable interest. C. speciosus has a wide range of therapeutic activities because of its phytochemicals: alkaloids, flavonoids, terpenoids, saponins, steroids, and phenolics, which possessed different pharmacological properties (Srivastava et al., 2011; Devi and Urooj, 2010; Verma and Khosa, 2009). 


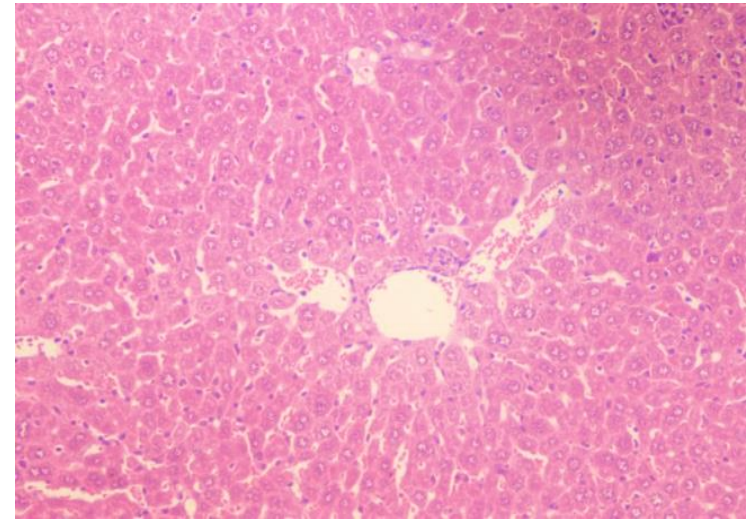

Figure 5A: A liver section from the normal group showed a central vein surrounded by the normal liver structure.

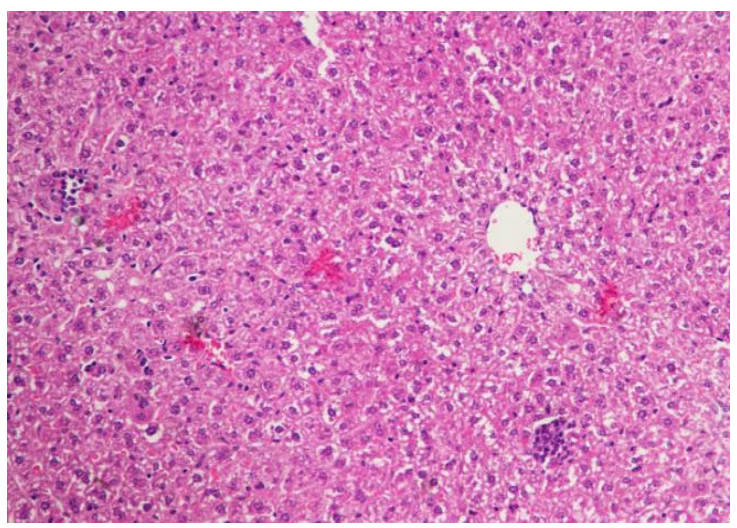

Figure 5C: A liver section from the treated group with $C$. speciosus $(100 \mathrm{mg} / \mathrm{kg})$ after PA-induced liver damage, demonstrated a mild improvement in the liver structure.

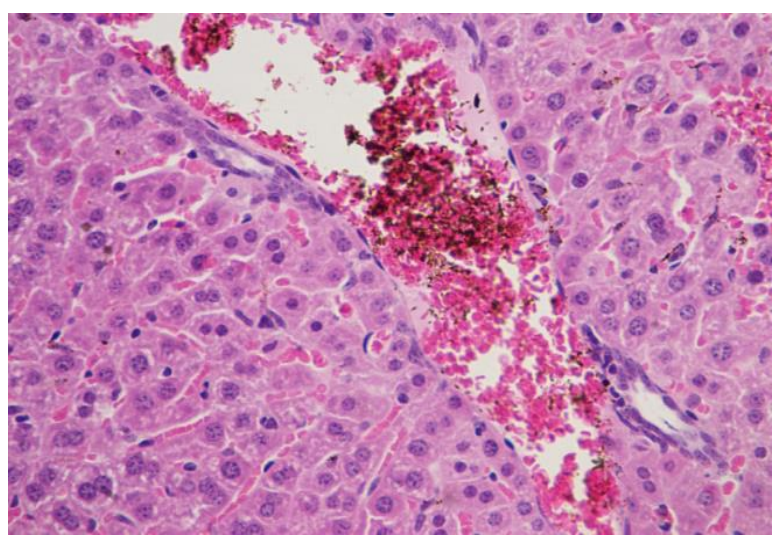

Figure 5B: A liver section from the PA group showed patches of liver cell congestion, necrosis, and apoptosis.

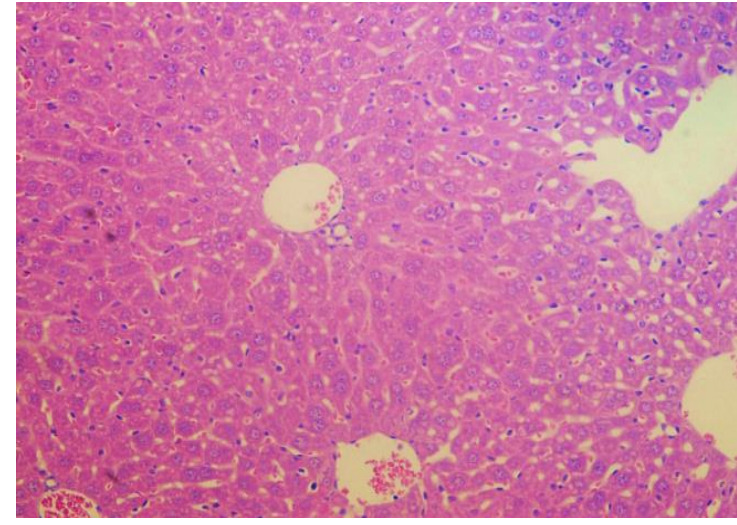

Figure 5D: A liver section from the treated group with C. speciosus $(200 \mathrm{mg} / \mathrm{kg})$ after PA-induced liver damage, showed a significant improvement in the liver structure more than that achieved using silymarin.

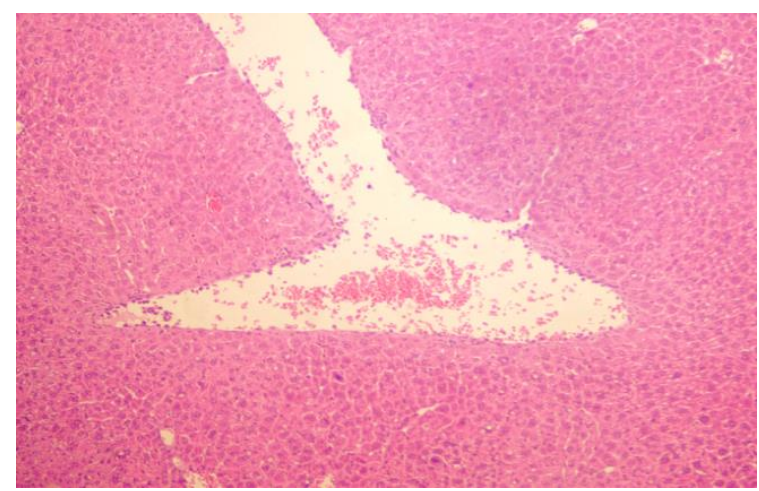

Figure 5E: A liver section from the treated group with silymarin (12 mg/kg b.w.) after PA-induced liver damage, showed an improvement in the liver structure; less than the treated group with C. speciosus (200 $\mathrm{mg} / \mathrm{kg}$ ).

It is worthy noted that only a few studies have focused on its hepatic influences. Hence, this study was designed to investigate the protective potential of $C$. speciosus towards the toxic effect of PA on the liver of normal healthy mice. PA is considered as self-poisoning worldwide due its wide availability and accessibility (Gunnell et al., 2000). Despite its safety when used properly, PA poisoning is one of the more common overdoses reported in poison centers (Heard, 2008). Normally, hepatoprotectant drugs preserve normal liver function or reduce the injurious effects caused by hepatotoxins ( $\mathrm{Li}$ et al., 2011). PA liver damage is produced from its metabolites, especially N-acetyl-pbenzoquinoneimine (NAPQI). NAPQI depletes the natural antioxidant, glutathione in the liver and consequently damages the hepatocytes, which finally leads to liver failure (Kulkarni, 2011). In the current study increased serum levels of ALT, AST, ALP, and TNF- $\alpha$ are the most important indicators of liver toxicity by PA (Kang, 2013). The obtained results revealed the protective effect of $C$. speciosus extract towards PA-induced hepatic damage. Also, they indicated that the level of serum marker enzymes and TNF- $\alpha$ decreased by increasing the dose of the MeOH extract, which proved its ability to save the hepatocytes from the damage that lead to secretion of ALT, AST, and ALP to the 
blood stream. In addition, no toxic changes in liver tissues were observed microscopically for the treated group by any dose of $C$. speciosus extract. This was in agreement with the previous study, which reported the protective potential of C. speciosus rhizomes EtOH extract towards $\mathrm{CCl}_{4}$-induced hepatotoxicity in rats (Verma and Khosa, 2009). It was stated that the hepatoprotective effect of $C$. speciosus could be attributed to the existence of steroidal saponins and different glycosides (Devi and Urooj, 2010; Verma and Khosa, 2009). Eremanthin and costunolide separated from $C$. speciosus rhizome reduced levels of ALT and AST in diabetic rats (Eliza et al., 2009a,b). Furthermore, it was reported that the alcoholic extract of $C$. speciosus rhizome had a potent antioxidant property which would help to reduce the oxidative stress and protect the liver from reactive oxygen species (Devi and Urooj, 2010; Eliza et al., 2010; Bhuyan and Zaman, 2008; Bavarva and Narasimhacharya, 2008). Thus, C. speciosus can be identified as a source of hepatoprotective compounds. This evidence further justifies the use of the alcoholic extract of $C$. speciosus as hepatoprotective agent. However, more extensive research should be conducted to confirm these effects in human.

\section{Conclusion}

The present investigation indicated that the $\mathrm{MeOH}$ extract of $C$. speciosus rhizomes provided significant hepatoprotective activity against PA-induced liver damage in mice. It produced a subsequent recovery near to normal level, which confirmed by the histopathological studies. The extract at a dose $200 \mathrm{mg} / \mathrm{kg}$ provided more significant reversal effect comparable with those of silymarin (reference standard). This hepatoprotection effect may be due to the active ingredients in $C$. speciosus, such as steroidal saponins and different glycosides as documented in the literature.

\section{Conflict of interest}

We wish to confirm that there are no known conflicts of interest associated with this publication and there has been no significant financial support for this work that could have influenced its outcome.

\section{References}

1. Akhila, A, Gupta, MM. and Thakur, R.S. (1987). Direct cyclisation of squalene to $5 \alpha-$ stigmast-9(11)-en-3 $\beta$-ol via $\Delta^{9(11)}$ lanosterol in Costus speciosus: a unique finding in sterol biosynthesis. Tetrahedron Lett. 28:4085-4088.

2. Al-Attas, A.A.M., El-Shaer, N.S., Mohamed, G.A., Ibrahim, S.R.M. and Esmat, A. (2015). Antiinflammatory sesquiterpenes from Costus speciosus rhizomes. J. Ethnopharmacol. 176: 365-374.

3. Banerji, R., Prakash, D., Patnaik, G.K. And Nigam, S.K. (1982). Spasmolytic activity of saponins. Indian Drugs. 20:51-54.

4. Bavarva, J.H. and Narasimhacharya, A.V.R.L. (2008). Antihyperglycemic and hypolipidemic effects of Costus speciosus in alloxan induced diabetic rats. Phytother. Res. 22:620-626.

5. Bhattacharya, S., Parikh, A., Debnath, P., Pandey, V. and Neogy, N. (1972). Anticholinesterase activity of Costus speciosus alkaloids. Indian J. Pharm.. 4:178-179.

6. Bhattacharya, S.K., Parikh, A.K., Debnath, P.K., Pandey, V.B. and Neogy NC. (1973). Pharmacological studies with the alkaloids of Costus speciosus. J. Res. Indian Med., 8:10-19.

7. Bhuyan, B. and Zaman, K. (2008). Evaluation of hepatoprotactive activity of rhizomes of Costus speciosus (J. Konig.) Smith. Pharmacologyonline. 3:119-126.

8. Chang, Y.Q., Tan, S.N., Yong, J.W.H. and Ge, L. (2012). Determination of flavonoids in Costus speciosus and Etlingera elatior by liquid chromatography-mass spectrometry. Anal. Lett. 45:345-355.

9. Chopra, R.N., Nayar, S.L., Chopra, I.C., Asolkar, L.V., Kakkar, K.K., Chakre, O.J. and Varma B.S. (2006). Glossary of Indian Medicinal Plants. (Including the Supplement). Council of Scientific and Industrial Research, New Delhi, pp. 176.

10. Cohen, S.D. and Khairallah, E.A. (1997). Selective protein arylation and acetaminophen-induced hepatotoxicity. Drug Metab. Rev. 29:59-77.

11. Devi, V. and Urooj, A. (2010). Nutrient Profile and Antioxidant components of Costus speciosus Sm. and Costus igneus Nak. Indian J. Nat. Prod. Resour. 1:116-118.

12. Eliza, J, Daisy, P. and Ignacimuthu S. (2010). Antioxidant activity of costunolide and eremanthin isolated from Costus speciosus (Koen ex. Retz) Sm. Chem. Biol. Interact. 188:467-472.

13. Eliza, J., Daisy, P., Ignacimuthu, S. and Duraipandiyan V. (2009b). Normo-glycemic and hypolipidemic effect of costunolide isolated from Costus speciosus (Koen ex. Retz.) Sm. in streptozotocin-induced diabetic rats. Chem. Biol. Interact. 179:329-334.

14. Eliza, J., Daisy, P., Ignacimuthu, S. and Duraipandiyan, V. (2009a). Antidiabetic and antilipidemic effect of eremanthin from Costus speciosus (Koen.) Sm., in STZ-induced diabetic rats. Chem. Biol. Interact. 182:67-72.

15. Elizabeth, M.W., David, T.O. and Fred, J.E. (1996). Selection, Preparation and Pharmacological Evaluation of Plant Material. John Wiley \& Sons Ltd, England, PP. 15-23.

16. Gunnell D., Murray V. and Hawton K. (2008). Use of paracetamol (acetaminophen) for suicide and nonfatal poisoning: worldwide patterns of use and misuse. Suicide Life Threat. Behav. 30:313-326. 
17. Gupta, M.M., Verma, R.K. and Akhila, A. (1986). Oxo acids and branched fatty acid esters from rhizomes of Costus speciosus. Phytochemistry, 25:1899-1902.

18. Gupta, R. (2010). Medicinal and aromatic plants. New Delhi: CBS Publishers \& Distributors, pp. 234-499.

19. Gupta, V.K. (2001). The wealth of India: first supplemented series (Raw materials). New Delhi: Council of Scientific New Delhi: Council of Scientific and Industrial Research, Pusa. 2:259-260.

20. Hazai, E., Monostory K., Bakos, A., Zacher, G. and Vereczkey, L. (2001). About hepatotoxicity of paracetamol overdose. Orv. Hetil. 42:345-349.

21. Heard, K.J. (2008). Acetylcysteine for acetaminophen poisoning. N. Engl. J. Med. 359:285-292.

22. Ibrahim, S.R.M., El-Shaer, N.S., Asfour, H.Z., Elshali, K.Z., Shaaban, M.I.A., Al-Attas, A.A.M. and Mohamed GA. (2017). Antimicrobial, antiquorum sensing, and antiproliferative activities of sesquiterpenes from Costus speciosus rhizomes. Pakistan J. Pharm. Sci. http://www.pjps.pk/?page_id=282.

23. Ichinose, K., You, S., Kawano, N., Hayashi, K., Yao, X. and Ebizuka, Y. (1999). Heterologous expression of furostanol glycoside 26-O- $\beta$-glucosidase of Costus speciosus in Nicotiana tabacum. Phytochemistry. 51:599603.

24. Inoue, K. and Ebizuka, Y. (1996). Purification and characterization of furostanol glycoside 26-O- $\beta$ glucosidase from Costus speciosus rhizomes. FEBS Lett. 378:157-160.

25. Jain, S. (1991). Dictionary of Indian folk medicine and ethnobotany. Deep Publications, New Delhi, pp.271.

26. Kang, K.S. (2013). Abnormality on liver function test. Pediatr. Gastroenterol. Hepatol. Nutr. 16:225-232.

27. Kulkarni, D. (2011). Acute paracetamol toxicity - A case report. Indian J. Appl. Res. 3:466-468.

28. Li, R., Guo, W., Fu, Z., Ding, G., Zou, Y. and Wang Z. (2011). Hepatoprotective action of radix Paeoniae Rubra aqueous extract against $\mathrm{CCl}_{4}$ induced hepatic damage. Molecules. 16:8684-8693.

29. Lijuan, W., Kupittayanant, P., Chudapongse, N., Wray, S. and Kupittayanant, S. (2011). The effects of wild ginger, Costus speciosus (Koen Smith) rhizome extract and diosgenin on rat uterine contractions. Reprod. Sci. 18:516-524.

30. Maheswari, C., Maryammal, R. and Venkatanarayanan, R. (2008). Hepatoprotective activity of Orthosiphon stamineus on liver damage caused by paracetamol in rats. Jordan J. Biol. Sci. 1:105-108.

31. Mahmood, A., Shukla, Y.N. and Thakur, R.S. (1984). Benzoquinones from Costus speciosus seeds. Phytochemistry. 23:1725-1727.

32. Singh, P., Mishra, G., Khosa, R., Srivastava, S., Jha, K. and Srivastava, S. (2011). Anthelmintic activity of aerial parts of Costus speciosus. Int. J. Green Pharm. 5:325-328.

33. Sivarajan, V.V. and Balachandran, I. (1994). Ayurvedic drugs and their plant sources. Oxford and IBH Publishing Co. Pvt. Ltd., New Delhi, pp. 439.

34. Srivastava, S., Singh, P., Jha, K.K., Mishra, G., Srivastava, S. and Khosa, R.L. (2013). Anti-inflammatory, analgesic and antipyretic activities of aerial parts of Costus speciosus Koen. Indian J. Pharm. Sci. 75:83-88.

35. Srivastava, S., Singh, P., Jha, K.K., Mishra, G., Srivastava, S. and Khosa, R.L. (2011). Costus speciosus (Keukand): A review. Der Pharmacia Sinica. 2:118-128.

36. Steinebrunner, N., Mogler, C., Vittas, S., Hoyler, B., Sandig, C., Stremmel, W. and Eisenbach C. (2014). Pharmacologic cholinesterase inhibition improves survival in acetaminophen-induced acute liver failure in the mouse. BMC Gastroenterol. 14:148.

37. Tewari, P.V., Chaturvedi, C. and Pandey, V.B. (1973). Antifertility activity of Costus speciosus. Indian J. Pharm. Sci. 35:114-115.

38. Verma, N. and Khosa, R.L. (2009). Evaluation of protective effect of ethanolic extract of Costus speciosus (Koen.) Sm. rhizomes of carbon tetrachloride induced hepatotoxicity in rats. Nat. Prod. Rad. 8:123-126.

39. Vermeulen, N.P.E., Bessems, J.G.M. and Vandestreat, R. (1992). Molecular aspects of paracetamol-induced hepatotoxicity and its mechanism-based prevention. Drug Metab. Rev. 24:367-407. 